Fecha de recepción: diciembre 2018 Fecha de aceptación: marzo 2019 Versión final: abril 2019

\section{Gerrit Rietveld y de Stijl: silla roja y azul, casa Schröder en Utrecht}

Martín Isidoro *

Resumen: Estas obras fueron realizadas en el marco del neoplasticismo o De Stijl, el cual se desarrolla entre 1917 y 1931 . Este movimiento hunde sus raíces estéticas en las críticas hechas al cubismo, considerado insuficientemente abstracto.

Pero, sus principios plásticos se forjan al contacto no sólo con las ideas neoplatónicas de Schoenmaekers sino también con los conceptos arquitectónicos de Wight y Belage. Y, aunque el nuevo camino estético haya sido abierto por la pintura, se tridimensionaliza -se hace realidad tangible de modo privilegiado- en el diseño de muebles y arquitectónico de Rietveld.

Palabras claves: De Stijl - Neoplasticismo - Gerrit Rietveld - silla roja y azul - casa Schröder

[Resúmenes en inglés y portugués en las páginas 71 - 72]

(*) Doctorando en Artes (UNLP). Docente de la Facultad de Filosofía y Letras (UBA). Investigador especializado en arte latinoamericano colonial. Lic en Historia del Arte (UBA).

\title{
Introducción
}

Estas obras fueron realizadas en el marco del neoplasticismo o De Stijl, el cual se desarrolla entre 1917 y 1931 . Este movimiento hunde sus raíces estéticas en las críticas hechas al cubismo, considerado insuficientemente abstracto. Pero, sus principios plásticos se forjan al contacto no solo con las ideas neoplatónicas de Schoenmaekers sino también con los conceptos arquitectónicos de Wight y Belage. Y, aunque el nuevo camino estético haya sido abierto por la pintura, se tridimensionaliza -se hace realidad tangible de modo privilegiado- en el diseño de muebles y arquitectónico de Rietveld.

\section{ABSTRACT}

Estas obras fueron realizadas en el marco del neoplasticismo o De Stijl, el cual se desarrolla entre 1917 y 1931. Su fecha y lugar de nacimiento oficial se ubica en el verano de 1917, en Laren, Holanda. (Jaffé, 1956, p. 45) Este movimiento hunde sus raíces estéticas en las críticas hechas al cubismo, considerado insuficientemente abstracto (Dempsey, 2002, p. 122). 
Pero, los principios plásticos y filosóficos del neoplasticismo se forjan al contacto, por un lado, con las ideas del filósofo neoplatónico Mathieu Schoenmaekers, y, por otro lado, con los conceptos arquitectónicos de Frank Lloyd Wight y Petrus Belage (Stangos, 1989, p. 122). En La nueva imagen del mundo de Schoenmaekers (1915), se define las nociones del dominio cósmico de la ortogonalidad y la del simbolismo de los colores primarios -de mezcla sustractiva-. Dichas nociones son principios básico de la estética neoplástica. En relación a la primera dice que:

Los dos completos opuestos fundamentales, que conforman nuestro planeta y todo lo terrenal, son: la línea de fuerza horizontal, es decir, la órbita terrestre alrededor del sol; y, el movimiento del rayo, vertical y profundamente espacial, que surge desde el centro del sol. (p. 102)

Schoenmaekers concibe al sistema de contradicciones como una parte primordial de su filosofía. Plantea que los contrarios están siempre relacionados uno con otro, de tal manera que pueden ser reducidos a la relación activo-pasivo. Por ejemplo, masculinidad-femineidad son contrarios, no opuestos. Y, en su sistema, formula otras series de ecuaciones, estrictamente paralelas: por un lado, vertical=espacio=evolución concreta; $y$, por el otro, horizontal=tiempo=historia concreta. Además, agrega que la línea absoluta caracteriza al tiempo absoluto, mientras que el rayo absoluto lo hace con el espacio absoluto. Así, formula un sistema dialéctico místico, donde los contrarios son aspectos diferentes de la misma realidad, son reales únicamente en función a su contraparte. Es decir, la línea es tal en función a la existencia del rayo, y viceversa. (Jaffé, 1956, p. 58)

Sobre este sistema místico de contrarios -de algún modo, abstruso-, Mondrian construye su teoría de oposición plástica, una de las piedras angulares de su doctrina. Para él, el pensamiento lógico demostró claramente una antigua verdad: una cosa dada puede sólo ser expresada o conocida por su contrario. (Jaffé, 1956, p. 58) En este punto, habría que considerar la influencia del concepto hegeliano de negatividad; por este motivo entre otros, Van Doesburg describe al neoplasticismo como la manifestación plástica de las leyes elaboradas por Hegel (Jaffé, 1956, p. 54).

... en toda realidad hay opuestos -y [o] también que dada una realidad, hay la opuesta a ella-. Pero entonces ni tal realidad ni la opuesta son realidades 'completas', pues una realidad sólo puede 'completarse' mediante los opuestos que la constituyen. Por tanto, según Hegel, en toda realidad hay alguna negación sin la cual no sería real. (Ferrater Mora, 1994, p. 2520)

Lo visible, la realidad concreta natural, no puede ser conocida por lo natural palpable, sino que por su contrario. Esto implica que la imagen de la realidad visible, puede sólo apelar a la consciencia presente del tiempo por medio de una expresión real-abstracta. (Jaffé, 1956, p. 58) Van Doesburg (1929, p. 628) cita las series de semejanzas en oposición de Mondrian: por una parte, vertical $=$ masculino=espacio=estático=armonía, etc.; $y$, por la otra parte, horizontal=femenino=tiempo=dinámico=melodía, etc. Y, luego, comenta que estos discretos medios de expresión, a pesar de su simpleza, tienen un contenido esencial, 
cósmico y vivaz, que para el hombre moderno significan más que una escultura de Fidias o Praxíteles.

Además, Schoenmaekers no sólo establece una diferencia cualitativa -no funcional- entre las líneas verticales y horizontales sino también hace una calificación mística de su intersección como símbolo. Su meditación sobre la construcción mística de la cruz -esto es, la confluencia del rayo y de la línea-, hace que vea la realidad como hecho creado: el movimiento vertical -rayo- y horizontal -línea- es cósmico, fuerza creativa, que se manifiesta a sí mismo en cualquier lugar, incluso en el más mínimo detalle de la realidad. De Stijl -sobre todo Mondrian- entiende la oposición del movimiento vertical y horizontal como un principio de la construcción natural, enfatizando la diferencia cualitativa entre dichos movimientos. Por ejemplo, Mondrian considera al rayo -hecho interno y, por ello, invisible- como una línea vertical en la creación plástica. (Jaffé, 1956, pp. 58-59)

Con respecto a la segunda noción de Schoenmaekers, el simbolismo de los colores primarios, señala que:

El color [...] es un fenómeno de un elemento visual: rayo, línea o centro plástico [es decir, la intersección de los dos primeros]. Los tres colores principales son amarillo, azul y rojo: en realidad son los únicos colores, porque todos los demás pueden reducirse a esos tres. Estos tres colores son fenómenos del movimiento puntual desde el rayo, la línea y lo plástico. En esos tres colores, vemos que la imagen completa se separa y se recrea. (Schoenmaekers, 1915, p. 224)

En relación al color amarillo, explica que

... es el movimiento del rayo. El color amarillo prístino es un color radiante; entre los colores, el amarillo es el más 'luminoso', se expande espacialmente, se despliega, 'salta hacia adelante', quiere ser el centro del movimiento espacial. (Schoenmaekers, 1915, p. 224)

En cuanto al azul, dice que

... es el color opuesto al amarillo. Como 'luz', el azul es naturalmente el movimiento espacial, pero como 'color' es contrario al amarillo fuerte; si el color es azul suave, flexible, da la impresión de que el amarillo se escurre, por detrás, alrededor del punto medio; como color es azul firmamento, línea, horizontalidad. (Schoenmaekers, 1915, pp. 224-225)

Con respecto al rojo, manifiesta que

... es el apareamiento de amarillo y azul. Una 'mezcla' de amarillo y azul da verde. Pero una unión viva e íntima de amarillo y azul da rojo. Rojo, no azul-rojo o amarillo-rojo sino rojo puro, es 'radiativo', el principio de la imagen completa, no se destaca como el amarillo, no cede como el azul, pero 'flota' en el espacio ante el ancho horizonte azul. (Schoenmaekers, 1915, p. 225) 
Y, esta simbología del color tiene también una correlación con los elementos visuales.

... el amarillo 'brilla', el azul 'retrocede y el rojo 'flota' [...] Ahora bien, esos tres verbos también pertenecen esencialmente a los tres elementos visuales: el espacio vertical brilla, la línea horizontal retrocede, y el centro plástico [movimiento de la línea y el rayo] es el punto flotante viviente, el punto de equilibrio vivo... (Schoenmaekers, 1915, p. 226)

Sin embargo, a este simbolismo de los colores primarios de Schoenmaekers, hay que confrontarlo con el sentido de oposición del color neoplástico de Mondrian y Van Dousburg. Mondrian comienza a distanciarse del cubismo hacia 1914 (Jaffé, 1956, p. 43), debido a que, para él, este movimiento no aceptaba las consecuencias lógicas de sus propios descubrimientos: el cubismo no desarrollaba la abstracción hasta su meta final, esto es, la expresión de la realidad pura. Mondrian creía que esta realidad sólo podía ser establecida a través de las plásticas puras, las cuales no están condicionadas por el sentimiento e ideas sujetivos. Había hecho un largo proceso de reflexión para descubrir que las formas particulares y el color natural evocaban estados subjetivos de sentimiento, que obscurecían la realidad pura. Pues, la apariencia natural de las formas cambia, mientras que la realidad permanece constante. Entonces, para crear una realidad pura plásticamente, consideraba que era necesario reducir no sólo las formas naturales a los elementos constantes de la forma sino también el color natural al color primario, con el fin de no crear otras formas y colores particulares con sus consecuentes limitaciones: esta purificación buscaba una mayor unidad. Este problema lo resolvió cuando tomó consciencia de que: por un lado, en las artes plásticas, la realidad puede ser expresada únicamente mediante el equilibrio del movimiento dinámico de la forma y el color; y, por otro lado, los medios puros proporcionan la manera más efectiva de lograrlo. Cuando el movimiento dinámico se establece a través de contrastes y oposiciones de los medios expresivos, las relaciones se convierten en la preocupación principal del artista, que está buscando crear el equilibrio. Así, el ángulo recto termina siendo la única relación constante y, que mediante las proporciones de su dimensión, se le puede dar movimiento a su expresión constante, es decir, hacerla vivir. Mientras que el color lo purifica, por una lado, volviendo el espacio blanco, negro o gris, y, por el otro, la forma, color primario, logrando la unidad. (Mondrian, 1942, pp. 10-11) Van Dousburg (1929, p. 626) plantea que una verdadera renovación del arte, de un nuevo plasticismo comienza cuando se cesa con el uso de los medios secundarios o auxiliares de expresión, esto es, la forma. Para la pintura, los medios de expresión primarios son los extremos opuestos del color, en estricta separación, en su más puro estado: rojo-gris, azul-negro, amarillo-blanco -siendo el primer elemento del par, positivo, y el segundo, negativo-. Además de este grupo de opuestos, los colores individuales forman las siguientes oposiciones: rojo-azul, azul-amarillo, amarillo-rojo. Por otra parte, la oposición de colores mezclados -naranja, violeta...- mitiga la fuerza energética, que caracteriza a todos los colores y, por ende, disminuye la tensión. Otros medios de expresión primarios en pintura son la oposición de espacio y localización -o bien, espacio y objeto-, como por ejemplo horizontal-vertical o tamaño-número. En esta conexión, la línea oblicua puede ser vista como la combinación de la horizontal y la vertical. Si se considera los colores positivos 
(rojo, azul, amarillo) como más y los negativos -gris, negro, blanco- como menos, y se los igualamos -es decir, se distribuye la tensión-, el resultado es un eje neutralizado, esto es: equilibrio. Esto sería esencialmente el factor constructivo en la nueva imagen, que no sólo es aplicable a la pintura neoplástica sino también a las otras artes.

Como en la pintura, los medios de expresión primarios en la escultura, arquitectura, música y poesía pueden sólo ser descubiertos en las últimas consecuencias, a saber, en la batalla final contra la forma. Los siguientes grupos de oposiciones pueden ser establecidas para la nueva arquitectura: superficie-masa, espacio-tiempo, material-resistencia, abierto-cerrado, construcción-contraconstrucción, estático-dinámico... Para la escultura: volumen-vacío, espacio tiempo, espacio-tamaño, superficial-profundo... Van Dousburg (1929, p. 626)

Se puede observar, hasta aquí, que De Stijl descansa sobre postulados espirituales o místicos, que contrastan con su racionalidad formal postcubista. Por otra parte, a este nuevo estilo concebido como un lenguaje plástico objetivo, abstracto, puro, hay que agregarle los fines prácticos de mejorar la sociedad.

Con la purificación y simplificación de todos los atributos accidentales de las artes, el neoplasticismo aspira a la realización de un universalismo legal y espiritual. El objetivo es exteriorizar mediante la pura expresión plástica la igualmente armonía pura, que el hombre afirmó haber encontrado en las leyes del cosmos. Por lo tanto, intenta hacer sujeto de la contemplación algo cercano a la Idea platónica: la representación de relaciones exactas y equilibradas, que logran o se acercan tanto como es posible al principio de equilibrio universal. Esto hizo que De Stijl se sintiera obligado a aplicar las nuevas verdades encontradas a todas las demás formas de actividad humana. Precisamente, este es origen de su carácter utópico. Entonces, el arte abstracto neoplástico tenía no sólo que servir de paradigma sino también enseñar a la humanidad cómo realizar esta misma armonía, aboliendo toda lo fortuito. Así, el neoplasticismo convierte un resultado estético en un principio ético. (Jaffé, 1956, p. 5)

Por otra parte, la arquitectura neoplástica suma un antecedente decisivo a su concepción espacial: la idea de una construcción homogénea wrightiana (Stangos, 1989, p. 122) u obra de arte total -Gesamtkunstwerk-. Frank Lloyd Wright contemplaba dentro de este concepto el diseño del mobiliario.

... es totalmente imposible considerar que el edificio es una cosa y su mobiliario otra, incluso su sitio y su ambiente aún otra. En el espíritu en que estos edificios se conciben, son todo una cosa, que debe preverse y mantenerse en la naturaleza de su estructura. Ellos son simples detalles estructurales de su carácter y completitud. Los aparatos de calefacción, los accesorios de iluminación, las mismas sillas y mesas, los armarios y los instrumentos musicales, donde sea viable, son parte del edificio. Ningún aparato o accesorio es admitido estrictamente como tal, donde las circunstancias permitan el desarrollo completo del esquema de construcción. (Wright, 1910, s/n) 
Por otra parte, era conciente que estaba fundando una nueva tradición de diseño integral, que no sólo hacía interactuar a cada objeto del edificio como parte del mismo sino también tenía en cuenta la idea de confort.

Entonces, hacer de una vivienda una obra de arte total: en sí misma expresiva y hermosa, y más íntimamente relacionada con la vida, que cualquier otra escultura o pintura independiente; dándose libre y adecuadamente a las necesidades individuales de los habitantes; una entidad armoniosa; ajustando el color, el patrón y la naturaleza de las utilidades; y, en sí mismo una expresión de ellas en carácter [...] Una vez logrado, esto se convertirá en una tradición, un gran paso hacia adelante con respecto al tiempo en que una vivienda se disponía como cuartos separados, meras habitaciones para contener un conjunto de muebles, sin comodidades. [...] Una entidad orgánica es esto, en contraste con esa adición: seguramente un ideal superior de unidad [...] Una cosa en lugar de muchas, una gran cosa en lugar de una colección de otras más pequeñas. (Wright, 1910, s/n)

De hecho, en arquitectura la disolución del movimiento se manifiesta: por un lado, con el alejamiento del concepto de la Gesamtkunstwerk; y, por el otro lado, en la aceptación de la introducción de objetos ajenos al diseño neoplástico, considerados como ready-made. (Stangos, 1989, p. 131)

¿Cómo vinculamos los pares categóricos morfológicos propuestos por esta investigación a los planteados por el neoplasticismo? Ambos se forjan entre el hecho empírico de las obras concretas observadas y la abstracción categorial sin querer caer en un formalismo; además, ambos plantean matices variables a partir de extremos. Sin embargo, entre ellos, básicamente, hay una diferencia óntica. Mientras que los primeros se originan como expresiones analíticas e historiográficas del diseño tridimensional, que buscan conceptualizar aspectos de la forma con carácter universal, los pares contrarios, no opuestos utilizados por De Stijl surgieron como resultado de la búsqueda de la constitución de un lenguaje artístico objetivo, moderno e, incluso, universal, que excluyera todo capricho individual. Una posible respuesta es pensar los pares categóricos de nuestra investigación a la luz de los resultados de la reflexión del sistema místico de contradicciones. Sin el contenido simbólico de los pares neoplásticos, toda lectura morfológica sería una lectura ingenua. Focalizándonos en las obras a analizar, comenzaremos con la silla roja y azul realizada en 1917 por Rietveld. Este mueble deriva tipológicamente de la silla-cama plegable victoriana, pero el vocabulario empleado está dado por la primera tridimensionalización de la estética neoplástica en las artes aplicadas. Es decir, emplea específicamente gran parte del esquema de colores canónicos: los primarios y el negro en la estructura lineal. Con esta silla se fundan las bases para una organización abierta del diseño espacial y, a la vez, libera al movimiento de la influencia estética de Wight, más allá de continuar con la idea de obra de arte total. (Stangos, 1989, p. 126) 


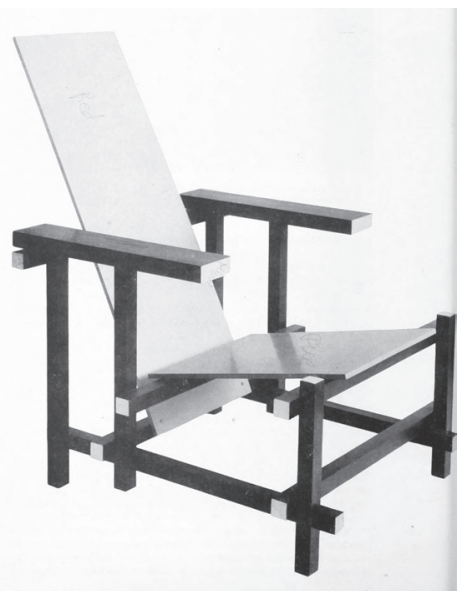

Figura 1.

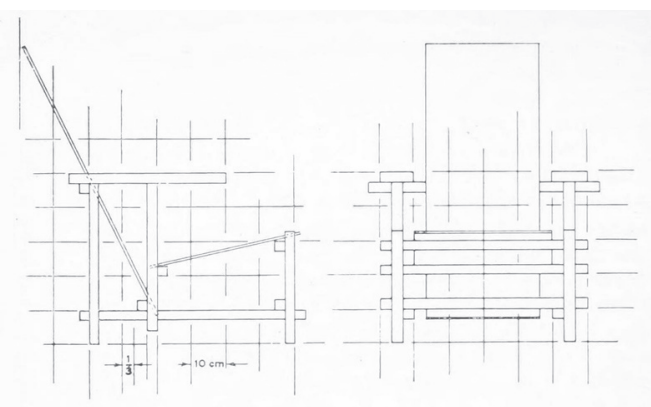

Figura 2.

Figura 1. Silla Roja y azul de Gerrit Rietveld, 1917. Fuente BROWN, T. (1958). Figura 2. Silla Roja y azul de Gerrit Rietveld, 1917 Fuente BROWN, T. (1958)

En relación a las categorías estructurales observables, en principio podemos hablar de una estructura definida por el empleo del color y la forma lineal neoplástica canónica. También, por la misma razón, hay que contemplar el polo unitario, ya que la hace parte del espacio neoplástico, concebido desde los mismos principios colorísticos y formales. Algunas de sus rasgos sobresalientes -ajustándonos a la propuesta de esta investigaciónson los polos dinámico, móvil, elástico y articulado, no sólo posibilitados por la tipología empleada sino también teniendo en cuenta la proyección de las ideas espaciales plasmadas en esta silla y, sobre todo, en la silla Berlín -1923- a la propuesta arquitectónica de la casa Schröder. Con respecto a las categorías texturales, se da de modo explícito el polo trasparente. Precisamente, Jaffé (1956, p. 164) explica que esta silla, por su transparencia, no obstruye visualmente el espacio y resuelve con esta característica el problema de la relación espacio-mobiliario, logrando de ese modo su integración total. Además, podríamos agregar que lo hace en la línea de la Gesamtkunstwerk, es decir, que estamos ante la presencia del polo homogéneo. Por otra parte, su diseño simple y puro nos da pie para considerar, en sentido amplio, el polo esquemático. Se podría incluso pensar este polo desde una máxima antidecorativa de De Stijl:

Sin decoración, nada que sea superfluo, nada que sea artístico en el sentido de acento en la belleza, agregado desde el exterior a posteriori. Sólo la sinceridad del objeto en sí mismo. Por encima de todo, verdad, función, construcción. En ningún lugar, un defecto debido a la reflexión individualista. (Van Doesburg, 1922, p. 36)

De hecho, este antidecorativismo purista llega a los extremos de la iconoclastia. 
A la pregunta de qué lugar ocupará la escultura en el nuevo interior, este mueble [silla roja y azul], a través de su nueva forma, da una respuesta: Nuestras sillas, mesas, armarios y otros utensilios son las imágenes -abstractas y realesde nuestro futuro interior. (Van Doesburg, 1919, p. 133)

En cuanto a esta casa, de 1924, situada en Utrecht -Países Bajos-, fue un proyecto de Rietveld en colaboración con la Sra. Trus Schröder-Schäder. Se caracteriza por: a) su asimetría; b) la articulación dinámica de sus partes y en su relación dentro-fuera; c) la explotación máxima de su interior a partir de la flexibilidad de sus paredes-tabique móviles, en el segundo nivel; d) zonas coloreadas que estructuran y modulan; e) los colores primarios y neutros antidecorativos cuya función es estructurar, acentuar, contrastar, articular, alejarse o flotar. Pero sobre todo, el espacio interior y el exterior en consecuencia -para el caso trabajado-, siguen la máxima de Van Doesburg que considera a la nueva arquitectura como una construcción de esqueleto abierto, que genera una espacialidad anticúbica y se dispersa centrífugamente desde el núcleo cúbico. Además, claro está, de ser una expresión acabada del concepto wrightiano de obra de arte total. (Stangos, 1989, pp. 129-130)

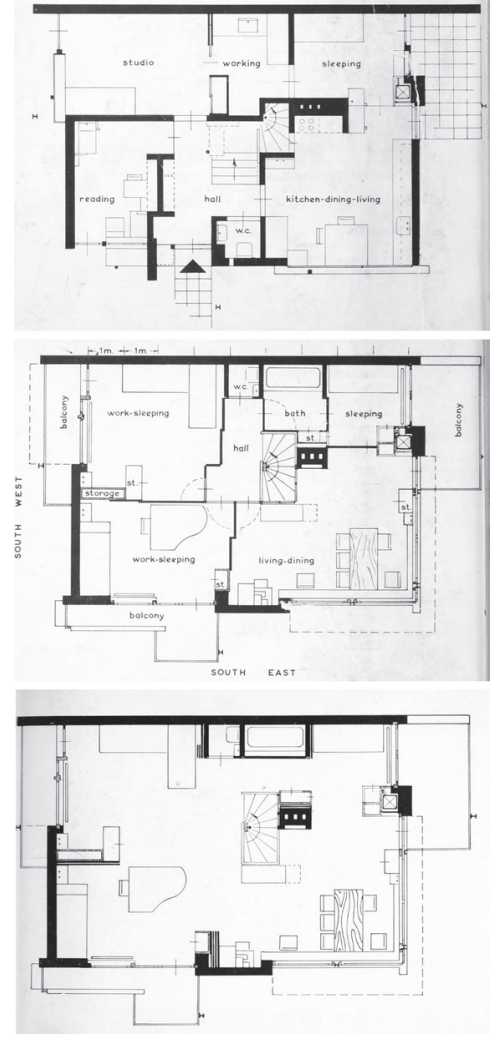

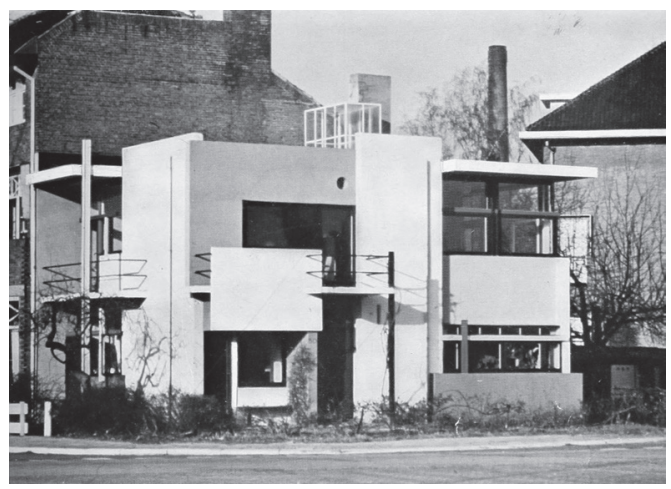

Figura 3.

Figura 3. Casa Schröder de Gerrit Rietveld, en Utrecht, 1924. Fuente: BROWN, T. (1958).

Figura 4. Casa Schröder de Gerrit Rietveld, planta inferior y superior -cerrada y abierta-. Fuente: BROWN, T. (1958)

Figura 4. 
En este punto, es necesario reflexionar sobre la importancia que Mondrian y Van Doesburg dan al espacio interior, durante la década del 20, en su proyecto utópico. Para ellos, este espacio jugará un rol decisivo, pues la creación de una nueva ciudad surgirá del nuevo hogar neoplástico, que no será un lugar de aislamiento o refugio sino parte de un todo urbano. Incluso Mondrian va más lejos en su pensamiento, afirmando que si las personas tuvieran diseñado sus interiores neoplásticamente, la pintura individual podría gradualmente desaparecer, ya que este estilo está más vivo cuando 'nos rodea'. (Jaffé, 1956, pp. 162-163) Desde nuestra perspectiva de análisis, las siguientes categorías texturales podrían tenerse en cuenta: homogéneo, diáfano, transparente-opaco en tensión, permeable-impermeable también en tensión. La homogeneidad se hace manifiesta entre el interior y el exterior que son uno, pues los voladizos parece surgir desde el interior y los solapamientos de los planos murarios exteriores no explicitan los límites precisos de la masa arquitectónica; también, las ventanas de esquina colaboran en tal efecto. Sin embargo, no sucede esto desde lo funcional.

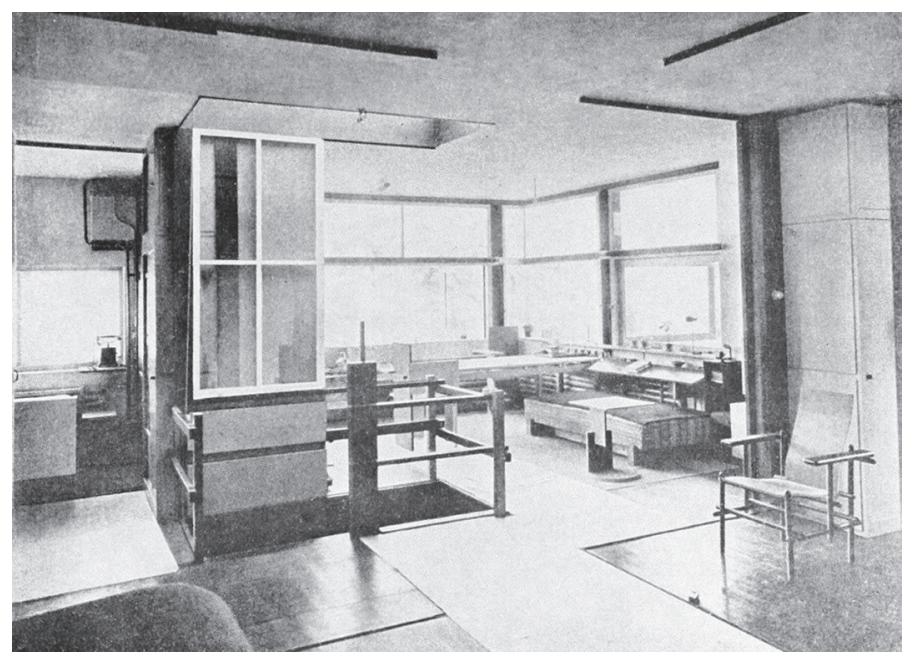

Figura 5. Casa Schröder, interior de la planta superior abierta. Fuente BROWN, T. (1958)

No existe evidencia de que Rietveld y la Sra. Schröder estudiaran los requerimientos funcionales -en este caso, las necesidades de una familia con tres hijos- y luego formulasen el programa arquitectónico estrictamente a partir de los factores funcionales. Más bien, establecieron arbitrariamente una variedad de diferentes tipos de volúmenes -cerrados, en el piso inferior; abiertos, en el superior- que podían ajustarse y adaptarse a las necesidades de cualquier habitante. El arquitecto pretendía más un diseño para una forma de vida, que un diseño para una familia específica. (Brown, 1958, p. 51) 
En cuanto a lo diáfano, este polo se desarrolla en la planta superior. Allí, planeles deslizables moldean el espacio tabicandolo o abriéndolo en función de las actividades que se realizarán.

En el momento en que diseñaron la casa, Rietveld y la Sra. Schröder estaban trabajando con la entonces revolucionaria idea de la planta abierta, aunque no operaron con un dogma consistente. Además, Rietveld deseaba deliberadamente este volumen en forma de cueva 'para aquellos animales que prefieren este tipo de madriguera'. (Brown, 1958, p. 47)

El par polar transparente-opaco lo encontramos en tensión en la estructura edilicia en sí, debido a que, aunque los aventanamientos abren marcadamente en algunos sectores el interior, todavía la presencia del muro es dominante -por este mismo motivo hallamos que el par permeable/impermeable también está tensión-. Sin embargo, el polo transparente se cumple en la distribución de los muebles integrados.

Los muebles y los gabinetes empotrados influencian el espacio, cortándolo sutilmente en segmentos más pequeños sin perjudicar su apertura. (Brown, 1958, p. 48)

En relación a la lectura tanto de la forma como del color desde los pares categóricos, es necesario sumar o, hacer directamente el análisis perceptivo desde las consecuencias simbólicas del sistemas de contrarios sobre el que se funda este estilo. No podemos, por ejemplo, en el color o en la forma, avanzar sobre el polo límite simplemente desde lo morfológico, sin atender a la simbología neoplástica, que percibe al amarillo como un color que 'irradia' superando sus límites o al rojo que 'flota' poniendo en crisis su ubicuidad perceptiva; así como el espacio vertical brilla o la línea horizontal retrocede, a pesar de que estas líneas pueden ser negras. Incluso, pensar en el par estático-dinámico para una estética utópica fundada en el principio ortogonal únicamente traería como resultado la segura elección del primer elemento de dicho par. Sin embargo, el movimiento en el neoplasticismo es místico y no puede escaparsenos a la percepción -que no hace falta recordar que es cultural y aprendida- que el movimiento vertical -rayo- y horizontal -línea- son cósmicos, y fuerza creativa en su intersección. A modo de ejemplo de lo planteado, partes del piso de la planta superior es rojo y también azul, es decir que no hay que dejar de pensar que al caminar sobre éste el espacio desde la percepción neoplástica 'flota' y 'se hunde', respectivamente.

A modo de conclusión, se pudo advertir que es totalmente viable un análisis morfológico, a través del empleo de pares categóricos, sin el riesgo de caer en formalismos. Por ello, hace tiempo que dentro del ámbito de la historia del arte se está reconsiderando críticamente el método formalista no sólo para corregir una visión estrecha y estereotipada del mismo sino también para repensar algunos aspetos teóricos y metodológicos viables en la investigación actual. ${ }^{1}$ Sin embargo, se hace necesario en una propuesta utópica de vanguardia comtemplar también su discurso al mismo nivel que lo observable empíricamente a partir del encuadre categorial. En el caso de De Stijl, la ideología y la producción plástica están íntimamente implicadas: Van Doesburg lo asume como expresiones equivalentes. 
(Jaffé, 1956, p. 54) De hecho, Mondrian afirmaba que

Mientras que la expresión espontánea, de la intuición, que logra una obra de arte -en otras palabras, su contenido espiritual- puede únicamente interpretarse por el arte de la palabra, aún queda la palabra sin el arte, un razonamiento, una explicación lógica, mediante la cual se puede demostrar la razonabilidad de una obra de arte. Por lo tanto, es posible que el artista de hoy hable de su propio arte. (Jaffé, 1956, p. 54)

\title{
Notas
}

1. Ver: Frank, M. y Adler, D.. (2016) German Art History and Scientific Thought: Beyond Formalism. New York: Routledge.

\section{Listas de Referencias Bibliográficas}

Brown, T. (1958) The work of G. Rietveld, architect. Utrecht: A. W. Bruna \&Zoon. Dempsey, A, (2002) Estilos, escuelas y movimientos. Buenos Aires: La Isla.

Ferrater Mora, J. (1994) Diccionario de filosofía. Barcelona: Ariel. Jaffé, H. (1956) De Stijl 1917-1931. The Dutch Contribution to Modern Art. Amsterdam: Meulenhoff. Recuprado de http://www.dbnl.org/tekst/jaff001stij01_01/colofon.htm Mondrian, P. (1942) Toward the true vision of reality. En: Mondrian, P. (1951) Plastic art and pure plastic art, 1937, and other essays, 1941-1943. New York: Wittenborn and company. Schoenmaekers, M. (1915) Het nieuwe wereldbeeld. Bussum: Van Dishoeck.

Stangos, N. (1989) Concepto de arte moderno. Madrid: Alianza Forma.

Van Doesburg, T. (1919) XXII. Aanteekeningen bij eén leunstoel Van Rietveld. En: De Stijl, Vol. 2, No 11, septiembre.

- (1922) Der Wille zum Stil. En: De Stijl, Vol. 5, № 33, marzo.

- (1929) Der Kampf um den neuen Stil. En: Neue Schweizer Rundschau. Recuperado de https://www.e-periodica.ch/digbib/view?pid=alp-004:1929:0::1101

Wright, F. (1910) Studies and Executed Buildings. Berlín: Wasmuth.

\begin{abstract}
These works were made within the framework of neoplasticism or De Stijl, that took place between 1917 and 1931. This movement has its aesthetic roots in the criticisms made to Cubism, considered insufficiently abstract. But, its plastic principles are forged in contact not only with the Neoplatonic ideas of Schoenmaekers but also with the architectural concepts of Wight and Belage. Although, the new aesthetic path has been opened by painting, it is three-dimensionalized - it becomes a tangible reality in a privileged way - in the furniture and architectural design of Rietveld.
\end{abstract}

Keywords: De Stijl - Neoplasticism - Gerrit Rietveld - red and blue chair - Schröder house. 
Resumo: Estas obras foram feitas no âmbito do neoplasticismo ou De Stijl, entre 1917 e 1931. Este movimento tem suas raízes estéticas nas críticas feitas ao cubismo, considerado insuficientemente abstrato. Mas, seus princípios plásticos são forjados em contato com as ideias neoplatônicas de Schoenmaekers, e também com os conceitos arquitetônicos de Wight e Belage. Embora o novo caminho estético tenha sido aberto pela pintura, ele é feito tridimensional - torna-se uma realidade tangível de maneira privilegiada - no mobiliário e no projeto arquitetônico de Rietveld.

Palavras chave: De Stijl - Neoplasticism - Gerrit Rietveld - cadeira vermelha e azul - casa Schröder

[Las traducciones de los abstracts fueron supervisadas por el autor de cada artículo] 